\title{
Results of the 2000 Creek Plantation Swamp Survey
}

by

P. D. Fledderman

Westinghouse Savannah River Company

Savannah River Site

Aiken, South Carolina 29808

DOE Contract No. DE-AC09-96SR18500

This paper was prepared in connection with work done under the above contract number with the U. S.

Department of Energy. By acceptance of this paper, the publisher and/or recipient acknowledges the U. S.

Government's right to retain a nonexclusive, royalty-free license in and to any copyright covering this paper, along with the right to reproduce and to authorize others to reproduce all or part of the copyrighted paper. 
This report was prepared as an account of work sponsored by an agency of the United States Government. Neither the United States Government nor any agency thereof, nor any of their employees, makes any warranty, express or implied, or assumes any legal liability or responsibility for the accuracy, completeness, or usefulness of any information, apparatus, product or process disclosed, or represents that its use would not infringe privately owned rights. Reference herein to any specific commercial product, process or service by trade name, trademark, manufacturer, or otherwise does not necessarily constitute or imply its endorsement, recommendation, or favoring by the United States Government or any agency thereof. The views and opinions of authors expressed herein do not necessarily state or reflect those of the United States Government or any agency thereof.

This report has been reproduced directly from the best available copy.

Available for sale to the public, in paper, from: U.S. Department of Commerce, National Technical Information Service, 5285 Port Royal Road, Springfield, VA 22161, phone: (800) 553-6847

fax: (703) 605-6900

email: orders@ntis.fedworld.gov

online ordering: http://www.ntis.gov/ordering.htm

Available electronically at http://www.doe.gov/bridge

Available for a processing fee to U.S. Department of Energy and its contractors, in paper, from: U.S. Department of Energy, Office of Scientific and Technical Information, P.O. Box 62, Oak Ridge, TN 37831-0062, phone: (865) 576-8401

fax: (865) 576-5728

email: reports@adonis.osti.gov 


\section{DISCLAIMER}

Portions of this document may be illegible in electronic image products. Images are produced from the best available original document. 
ESH-EMS-2000-547

Rev. 0

May 31, 2000

\section{Results of the 2000 Creek Plantation Swamp Survey}

P.D. Fledderman

Environmental Monitoring Section

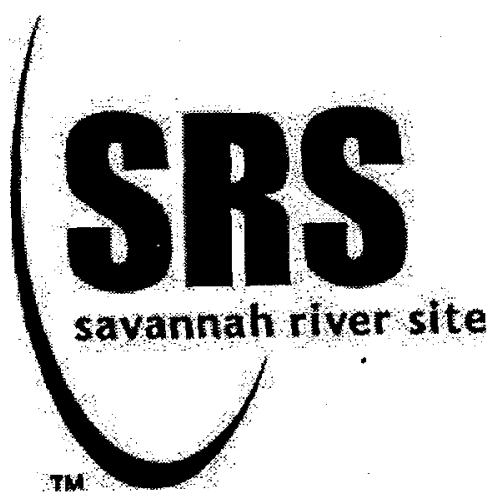




\section{INTRODUCTION}

The Creek Plantation is a privately owned land area that is located along the Savannah River and borders the southeast portion of SRS. The land is primarily undeveloped and agricultural; its purpose is to engage in equestrian-related operations. A portion of Creek Plantation along the Savannah River is a low-lying swamp, known as the Savannah River Swamp, which is uninhabited and not easily accessible.

In the 1960s, an area of the Savannah River Swamp on Creek Plantation- specifically, the area between Steel Creek Landing and Little Hell Landing - was contaminated by SRS operations. Failed experimental fuel elements leaked activity into the P-Area storage basin, whose water occasionally was discharged to Steel Creek. During high river levels, water from Steel Creek flowed along the lowlands comprising the swamp, resulting in deposition of radioactive material. This water eventually discharged to the Savannah River at Little Hell Landing, resulting in the contamination of a portion of the Savannah River Swamp.

In 1974, SRS studies estimated that a total of approximately $25 \mathrm{Ci} \mathrm{Cs}-137$ and $1 \mathrm{Ci} \mathrm{Co}-60$ were deposited in the swamp. Following this discovery, a series of surveys was initiated to characterize the contaminated environment. These surveys evolved into a long-term surveillance program, with surveys conducted periodically to monitor the extent and level of contamination present. Table 1 presents an overview of the surveys conducted since 1974. A detailed summary of these results is in the preparation stage.

During the early surveys, a series of 10 sampling trails was established through the swamp, ranging from 240 to 3,200 feet in length. Fifty-two monitoring locations were designated on the trails to allow for continued monitoring at a consistent set of locations.

\section{SURVEY DETAILS}

The latest survey was scheduled for late 1999 , but hunting in the swamp area delayed the start until early 2000. The 2000 survey was identified as a comprehensive survey, requiring extensive media sampling and analysis- as well as exposure rate measurement. Tables 2 and 3 present a summary of the 2000 survey sampling and analysis plan.

The 2000 survey was conducted from early January through late April. Because of adverse field conditions, not all scheduled samples could be obtained. High water levels in January prevented sample collection at four locations, while the lack of green grass-type vegetation prevented collection of vegetation samples at an additional 38 locations.

\section{ANALYTICAL RESULTS}

Analytical results are presented in table 4 . With one exception (a sample was accidentally discarded prior to completion), all samples were successfully analyzed. As anticipated, based on source term information and historical survey results, Cs-137 was the primary radionuclide detected. Smaller numbers of samples showed low levels of $\mathrm{Co}-60$ and/or total strontium.

Cesium-137 was detected in 67 of the 71 soil samples and seven of the 11 vegetation samples. Cesium-137 concentrations in soil varied from nondetectable to $102 \mathrm{pCi} / \mathrm{g}$, while Cs-137 concentrations in vegetation varied from nondetectable to $4.4 \mathrm{pCi} / \mathrm{g}$. These observed concentrations are consistent with historical results. In general, higher levels of Cs-137 in soil were observed in the trails closer to the SRS boundary, although somewhat elevated levels in soil were observed as far as approximately four miles away (trail 9). All vegetation samples with detectable activity were collected from trails 1 and 2 . The samples with higher concentrations were located in the interior portions of each trail; this was expected based on the initial deposition mechanism and on previous survey results. As previously observed, the vertical distribution profile in soil is not as pronounced as it is in undisturbed areas. This indicates some movement (mobilization, movement and/orredeposition) of contamination in the swamp. No elevated Cs-137 levels were observed in samples from trail 10 , indicating that the area of contamination has not spread beyond the current survey area boundary. 
Cobalt- 60 was detected in only seven of the 71 soil samples and none of the vegetation samples. The Co- 60 concentrations in soil varied from nondetectable to $0.3 \mathrm{pCi} / \mathrm{g}$. These concentrations were consistent with historical survey results. Although no definite spatial distribution was observed, the sites with detectable Co- 60 concentrations generally were at locations showing relatively high Cs-137 concentrations. As with the distribution of Cs-137 activity, this trend was expected based on the initial deposition mechanism and on previous survey results.

Total strontium was detected in five of the 29 soil samples selected for analysis; no vegetation samples were analyzed for total strontium. Although slightly increased, these levels generally were consistent with historical survey results. Changes in the analytical method used may be partly responsible for the increase. Although the samples showing detectable activity were core segments, no apparent spatial distribution or correlation with Cs-137 concentrations was observed.

Thermoluminescent dosimeters (TLD) sets were placed at 50 of the 54 monitoring sites to determine ambient gamma exposure rates. Forty-eight of the 50 sets were retrieved from the swamp; the exposure time varied from 56 to 98 days. The gamma exposure rate ranged from 0.26 to $0.81 \mathrm{mrem} /$ day, which is consistent with the range observed in the 1993 survey - the most recent in which exposure rates were determined. The highest exposure rates were measured on trails 4 and 5 . In general, areas of higher exposure rates showed elevated Cs-137 concentrations; however, the locations of highest exposure rates do not directly correspond with the locations that have the highest Cs-137 activity. This is not unexpected, since each measured Cs-137 level is the concentration at a single point, while the TLD has a "field of view," which integrates exposure over an entire area.

\section{CONCLUSION}

The results of the 2000 survey of the Creek Plantation swamp generally were consistent with those observed in previous surveys. Over time, some changes in the spatial distribution of activity throughout the swamp has been observed, which means that some localized movement of activity may be occurring. However, there has been little change in the results from the downstream location (trail 10), which indicates that activity is not migrating out of the identified contaminated area. 
TABLE 1

Historical Survey Summary

\begin{tabular}{|c|c|c|}
\hline YEAR & SURVEY TYPE & MEDLA \\
\hline 1974 & Comprehensive & Soil, Vegetation, Exposure \\
\hline 1975 & Comprehensive & Soil, Vegetation, Exposure \\
\hline 1976 & Comprehensive & Soil, Vegetation, Exposure \\
\hline 1977 & Comprehensive & Soil, Vegetation, Exposure \\
\hline 1978 & Cursory & Exposure \\
\hline 1979 & Cursory & Exposure \\
\hline 1980 & Cursory & Exposure \\
\hline 1981 & Cursory & Exposure \\
\hline 1982 & Comprehensive & Soil, Vegetation, Exposure \\
\hline 1983 & Cursory & Exposure \\
\hline 1984 & Cursory & Exposure \\
\hline 1985 & Comprehensive & Soil, Vegetation, Exposure \\
\hline 1986 & Cursory & Soil, Vegetation, Exposure \\
\hline 1987 & Cursory & Soil, Vegetation, Exposure \\
\hline 1988 & Cursory & Soil, Vegetation, Exposure \\
\hline 1989 & Cursory & Soil, Vegetation, Exposure \\
\hline 1990 & Comprehensive & Soil, Vegetation, Exposure \\
\hline 1991 & Cursory & Soil, Vegetation, Exposure \\
\hline 1992 & Cursory $^{2}$ & N/A \\
\hline 1993 & Cursory & Soil, Vegetation, Exposure \\
\hline 1994 & Cursory & Soil, Vegetation \\
\hline 1995 & Not Conducted & N/A \\
\hline 1996 & Comprehensive & Soil, Vegetation \\
\hline 1997 & Not Conducted & N/A \\
\hline 1998 & Not Conducted & N/A \\
\hline 1999 & Not Conducted & N/A \\
\hline 2000 & Comprehensive & Soil, Vegetation, Exposure \\
\hline
\end{tabular}

NOTES:

${ }^{1}$ The meaning of "comprehensive" and "cursory" surveys has changed with time. Generally, comprehensive surveys involve extensive sampling with detailed analyses, while cursory surveys involve a more limited level of sampling and analyses.

${ }^{2}$ The survey was initiated but not completed because of continued high water. 
TABLE 2

2000 Survey Sampling and Analytical Details

\begin{tabular}{|l|l|l|l|}
\hline Matrix & \multicolumn{1}{|c|}{ Locations } & \multicolumn{1}{c|}{ Details } & \multicolumn{1}{c|}{ Analyses } \\
\hline Soil & $\begin{array}{l}\text { 10 sampling trails (54 } \\
\text { locations, see below) }\end{array}$ & $\begin{array}{l}0-3 " \text { samples at each } \\
\text { location } \\
0 "-3 ", 3 "-6 ", 6 "-9 ", \\
\text { and 9"-12" samples at } \\
\text { one location per trail }\end{array}$ & Gamma spec \\
\hline Vegetation & N/A & Gamma spec., total Sr \\
\hline $\begin{array}{l}\text { Ambient gamma } \\
\text { radiation }\end{array}$ & $\begin{array}{l}\text { Same as soil } \\
\text { Each media sampling } \\
\text { below) }\end{array}$ & N/A locations, see & Place TLDs \\
\hline
\end{tabular}

TABLE 3

2000 Survey Sampling Location Matrix

\begin{tabular}{|c|c|c|c|c|c|c|c|c|c|}
\hline Trail & River Mile & \multicolumn{7}{|c|}{ Sampling Points (feet from Savannah River) } \\
\hline 1 & 141.5 & 0 & 585 & 1175 & 1805 & $\mathbf{2 1 5 0}$ & 2640 & & \\
\hline 2 & 140.8 & 0 & 680 & 1330 & 1960 & 2620 & $\mathbf{3 1 0 0}$ & 3200 & \\
\hline 3 & $139.5-140.8$ & 0 & $\mathbf{9 2 0}$ & 2055 & & & & & \\
\hline 4 & 139.0 & 0 & 960 & 1245 & 1690 & $\mathbf{1 9 0 0}$ & 2390 & & \\
\hline 5 & 138.5 & 0 & 1750 & 1880 & $\mathbf{2 1 0 0}$ & 2535 & & & \\
\hline 6 & 137.0 & 0 & 1800 & 2300 & $\mathbf{2 5 3 0}$ & 2680 & & & \\
\hline 7 & 136.5 & 0 & 1900 & 2600 & $\mathbf{2 7 0 0}$ & 3100 & 3200 & & \\
\hline 8 & 135.7 & 0 & 550 & 915 & $\mathbf{1 4 6 0}$ & 2005 & 2670 & $\mathbf{2 9 0 0}$ & 3000 \\
\hline 9 & 135.5 & 0 & 1680 & 2035 & $\mathbf{2 2 0 0}$ & 2525 & & & \\
\hline 10 & 134.4 & 0 & $\mathbf{1 0 0}$ & 240 & & & & & \\
\hline
\end{tabular}

NOTE:

Locations in bold represent the site on each trail with the highest activity historically. Sampling at these locations will include 1 -foot core samples segmented into 3 -inch increments. 
TABLE 4

2000 Creek Plantation Survey Analytical Results

\begin{tabular}{|c|c|c|c|c|c|c|c|c|c|c|c|c|}
\hline Trail & $\begin{array}{c}\text { Distance } \\
\text { from } \\
\text { River }(\mathrm{ft})\end{array}$ & Media & $\begin{array}{l}\text { Sample ID } \\
\text { Number }\end{array}$ & $\begin{array}{c}\text { Collection } \\
\text { Date }\end{array}$ & $\begin{array}{l}\text { Depth } \\
\text { (inch) }\end{array}$ & $\begin{array}{l}\text { Cs-137 } \\
\text { (pCi/g) }\end{array}$ & $\begin{array}{l}\text { Cs-137 } \\
\text { Error }\end{array}$ & $\begin{array}{c}\mathrm{Co}-60 \\
(\mathrm{pCi} / \mathrm{g})\end{array}$ & $\begin{array}{l}\text { Co-60 } \\
\text { Error }\end{array}$ & $\begin{array}{c}\mathrm{Sr}-89,90 \\
(\mathrm{pCi} / \mathrm{g})\end{array}$ & $\begin{array}{c}\text { Sr-89,90 } \\
\text { Error }\end{array}$ & $\begin{array}{c}\text { Gamma } \\
\text { Exposure } \\
\text { (mrem/d) }\end{array}$ \\
\hline 1 & 0 & $S$ & 0081594700 & $01 / 13 / 00$ & $0-3$ & $5.87 \mathrm{E}+00$ & $3.16 \mathrm{E}-01$ & $4.08 \mathrm{E}-02$ & $2.77 \mathrm{E}-02$ & $\mathrm{~N} / \mathrm{A}$ & $\mathrm{N} / \mathrm{A}$ & 0.40 \\
\hline 1 & 0 & $\mathrm{~V}$ & $\mathrm{~N} / \mathrm{C}$ & & & & & & & & & \\
\hline 1 & 585 & $S$ & 0081595000 & $01 / 13 / 00$ & $0-3$ & $8.94 \mathrm{E}+00$ & $4.66 \mathrm{E}-01$ & $7.05 \mathrm{E}-02$ & $3.14 \mathrm{E}-02$ & $\mathrm{~N} / \mathrm{A}$ & $\mathrm{N} / \mathrm{A}$ & 0.39 \\
\hline 1 & 585 & $\mathrm{~V}$. & 0081598100 & $01 / 13 / 00$ & & $4.06 \mathrm{E}-01$ & $8.07 \mathrm{E}-02$ & $5.25 \mathrm{E}-02$ & $3.45 \mathrm{E}-02$ & $\mathrm{~N} / \mathrm{A}$ & $\mathrm{N} / \mathrm{A}$ & \\
\hline 1 & 1175 & $S$ & 0081594900 & $01 / 13 / 00$ & $0-3$ & $1.86 \mathrm{E}+01$ & $9.36 \mathrm{E}-01$ & $7.89 \mathrm{E}-02$ & $3.55 \mathrm{E}-02$ & $\mathrm{~N} / \mathrm{A}$ & N/A & 0.47 \\
\hline 1 & 1175 & $\mathrm{~V}$ & $\mathrm{~N} / \mathrm{C}$ & & & & & & & & & \\
\hline 1 & 1805 & $S$ & 0081594600 & $01 / 13 / 00$ & $0-3$ & $8.49 \mathrm{E}+01$ & $2.98 \mathrm{E}+00$ & $3.18 \mathrm{E}-01$ & $2.54 \mathrm{E}-02$ & N/A & $\mathrm{N} / \mathrm{A}$ & 0.66 \\
\hline 1 & 1805 & $\mathrm{~V}$ & 0081597900 & $01 / 13 / 00$ & & $2.34 \mathrm{E}+00$ & $1.46 \mathrm{E}-01$ & $-8.23 \mathrm{E}-03$ & $2.50 \mathrm{E}-02$ & $\mathrm{~N} / \mathrm{A}$ & $\mathrm{N} / \mathrm{A}$ & \\
\hline 1 & 2150 & $S$ & 0081597500 & $01 / 13 / 00$ & $0-3$ & $9.30 \mathrm{E}+01$ & $3.27 \mathrm{E}+00$ & $2.77 \mathrm{E}-01$ & $2.64 \mathrm{E}-02$ & $1.15 \mathrm{E}-01$ & $9.49 \mathrm{E}-02$ & 0.69 \\
\hline 1 & 2150 & $\mathrm{~S}$ & 0081597600 & $01 / 13 / 00$ & $3-6$ & $2.77 \mathrm{E}+01$ & $1.38 \mathrm{E}+00$ & $6.28 \mathrm{E}-02$ & $2.88 \mathrm{E}-02$ & $2.38 \mathrm{E}-02$ & $1.29 \mathrm{E}-01$ & \\
\hline 1 & 2150 & $S$ & 0081597700 & $01 / 13 / 00$ & $6-9$ & $7.85 \mathrm{E}+00$ & $4.09 \mathrm{E}-01$ & $6.48 \mathrm{E}-02$ & $3.14 \mathrm{E}-02$ & $2.35 \mathrm{E}-01$ & $1.40 \mathrm{E}-01$ & \\
\hline 1 & 2150 & $S$ & 0081597800 & $01 / 13 / 00$ & $9-12$ & $1.54 \mathrm{E}+00$ & $9.64 \mathrm{E}-01$ & $-3.74 \mathrm{E}-02$ & $1.35 \mathrm{E}-02$ & $1.62 \mathrm{E}-01$ & $1.35 \mathrm{E}-01$ & \\
\hline 1 & 2150 & $\mathrm{~V}$ & $\mathrm{~N} / \mathrm{C}$ & & & & & & & & & \\
\hline 1 & 2640 & $S$ & 0081594800 & $01 / 13 / 00$ & $0-3$ & $8.59 \mathrm{E}-01$ & $6.78 \mathrm{E}-02$ & $1.83 \mathrm{E}-02$ & $1.74 \mathrm{E}-02$ & $\mathrm{~N} / \mathrm{A}$ & N/A & 0.28 \\
\hline 1 & 2640 & $\mathrm{~V}$ & 0081598000 & $01 / 13 / 00$ & & $6.14 \mathrm{E}-02$ & $2.03 \mathrm{E}-02$ & $2.37 \mathrm{E}-02$ & $1.99 \mathrm{E}-02$ & $\mathrm{~N} / \mathrm{A}$ & N/A & \\
\hline
\end{tabular}




\begin{tabular}{|c|c|c|c|c|c|c|c|c|c|c|c|c|c|c|c|c|c|c|c|c|c|c|c|c|c|}
\hline 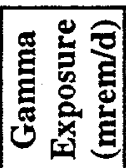 & & & 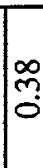 & & $\sqrt[3]{0}$ & & గ్య & & $\overrightarrow{0}$ & & \begin{tabular}{l}
$\infty$ \\
\multirow{0}{0}{} \\
0
\end{tabular} & & & & & & & 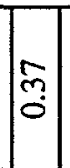 & & ڤ్ & & & & & \\
\hline 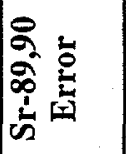 & $\frac{\varangle}{z}$ & & $\frac{\mathbb{z}}{\mathrm{z}}$ & $\frac{\varangle}{z}$ & $\frac{\$}{\mathrm{z}}$ & $\frac{\varangle}{z}$ & $\frac{\varangle}{z}$ & $\frac{\varangle}{z}$ & $\frac{\varangle}{z}$ & & 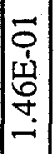 & 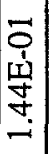 & 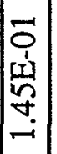 & 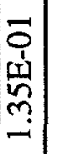 & & & $\frac{\$}{z}$ & $\bar{z}$ & & 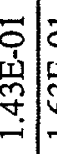 & 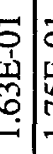 & 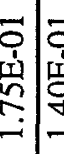 & & $\frac{\$}{z}$ & $\frac{\varangle}{z}$ \\
\hline 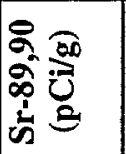 & $\frac{4}{z}$ & & $z$ & $\frac{\leqslant}{z}$ & $\frac{\Sigma}{z}$ & $\bar{z}$ & $\begin{array}{l}\varangle \\
\mathrm{z}\end{array}$ & $\frac{\varangle}{z}$ & $\mid \begin{array}{l}\varangle \\
z\end{array}$ & & 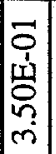 & 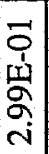 & 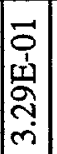 & 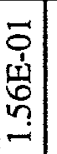 & & & $\frac{\varangle}{\mathrm{z}}$ & 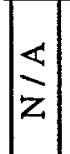 & & & 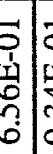 & 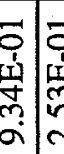 & & $\frac{\varangle}{z}$ & $\frac{\ll}{z}$ \\
\hline 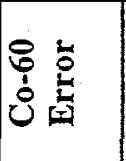 & $\mid \begin{array}{c}5 \\
0 \\
1 \\
2 \\
2 \\
-\end{array}$ & & 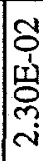 & 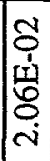 & 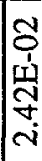 & 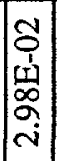 & 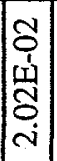 & 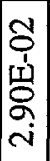 & 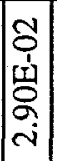 & & 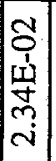 & 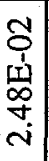 & 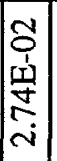 & 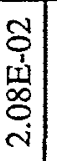 & 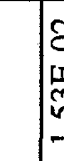 & 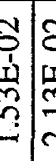 & 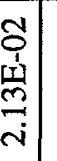 & 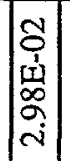 & & & 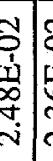 & 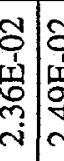 & & 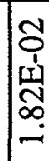 & 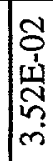 \\
\hline 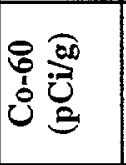 & 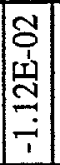 & & 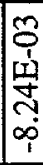 & 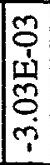 & 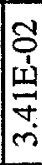 & 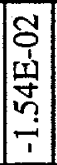 & $\left|\begin{array}{c}0 \\
0 \\
1 \\
1 \\
0 \\
0 \\
\sim\end{array}\right|$ & 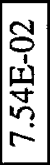 & $\left|\begin{array}{c}\delta \\
0 \\
1 \\
\\
\hdashline \\
\dot{+}\end{array}\right|$ & & $\left|\begin{array}{c}1 \\
0 \\
1 \\
\infty \\
0 \\
0 \\
0\end{array}\right|$ & 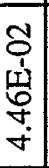 & \begin{tabular}{|c|} 
\\
0 \\
\\
$\vdots$ \\
0 \\
-1 \\
\end{tabular} & 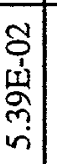 & & 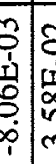 & 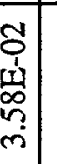 & $\mid$\begin{tabular}{c}
0 \\
0 \\
0 \\
0 \\
0 \\
\hdashline \\
\hdashline \\
\end{tabular} & & 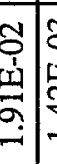 & 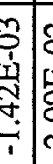 & 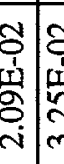 & & 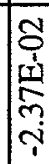 & $\begin{array}{l}m \\
0 \\
1 \\
0 \\
0 \\
0 \\
\infty \\
1\end{array}$ \\
\hline 蒙 & 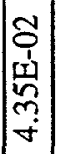 & & 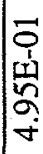 & $\begin{array}{c}\mathcal{O} \\
0 \\
1 \\
1 \\
\\
\infty \\
-1\end{array}$ & 要 & 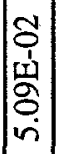 & $\left|\begin{array}{c}0 \\
0 \\
\dot{1} \\
0 \\
0 \\
0 \\
0\end{array}\right|$ & 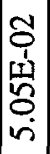 & 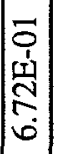 & & $\left|\begin{array}{c}8 \\
+ \\
+ \\
\infty \\
= \\
-\end{array}\right|$ & $\begin{array}{c}\overline{0} \\
0 \\
0 \\
0 \\
0 \\
\sim\end{array}$ & 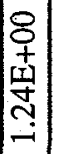 & 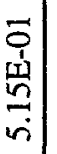 & & 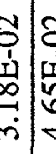 & 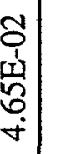 & 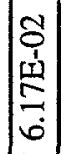 & & 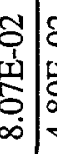 & 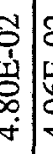 & 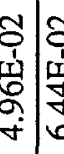 & & 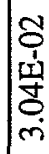 & 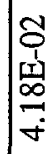 \\
\hline 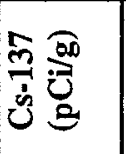 & 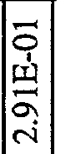 & & 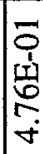 & $\begin{array}{c}9 \\
8 \\
1 \\
n \\
m \\
m \\
m \\
m\end{array}$ & $\begin{array}{l}0 \\
0 \\
\text { जी } \\
2 \\
\text { is }\end{array}$ & 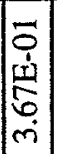 & $\mid \begin{array}{c}0 \\
0 \\
1 \\
0 \\
0 \\
0 \\
0\end{array}$ & $\begin{array}{l}\overline{0} \\
1 \\
\vdots \\
0 \\
ن \\
ن\end{array}$ & 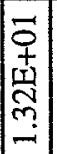 & & 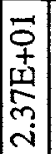 & 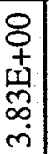 & $\left|\begin{array}{c}0 \\
0 \\
+ \\
0 \\
0 \\
i \\
\text { s. }\end{array}\right|$ & 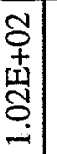 & & 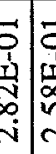 & $\begin{array}{c}-1 \\
0 \\
1 \\
0 \\
\infty \\
\vdots \\
\end{array}$ & $\left|\begin{array}{c}\sigma \\
0 \\
1 \\
\infty \\
\infty \\
\sigma \\
\dot{\gamma}\end{array}\right|$ & & \begin{tabular}{l|l}
8 & \\
+ & \\
0 & 0 \\
0 & 0 \\
\hdashline & 0
\end{tabular} & 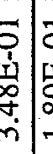 & 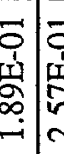 & & 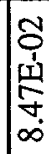 & 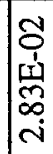 \\
\hline 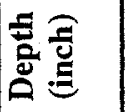 & $\hat{o}$ & & $\hat{o}$ & & $\hat{d}$ & & के & & $\dot{\delta}$ & & $\hat{o}$ & $\begin{array}{l}1 \\
1 \\
m\end{array}$ & $\left|\begin{array}{l}a \\
b\end{array}\right|$ & $\frac{N}{\alpha}$ & & $\hat{b}$ & & $\hat{o}$ & & c) & $\begin{array}{l}0 \\
1 \\
m\end{array}$ & $\grave{b}$ & & $\hat{\delta}$ & \\
\hline 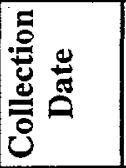 & $\mid$\begin{tabular}{c}
8 \\
8 \\
\hdashline \\
$\vdots$ \\
0
\end{tabular} & 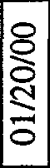 & $\mid \begin{array}{l}8 \\
8 \\
\\
0\end{array}$ & 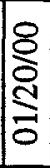 & 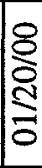 & $\mid$\begin{tabular}{c}
8 \\
$\stackrel{8}{8}$ \\
\hdashline \\
0
\end{tabular} & 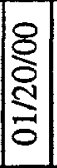 & $\begin{array}{l}\frac{8}{\delta} \\
\stackrel{\oplus}{\delta} \\
0\end{array}$ & 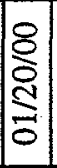 & & $\left|\begin{array}{c}8 \\
8 \\
0 \\
0 \\
0\end{array}\right|$ & 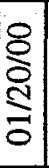 & $\left|\begin{array}{c}8 \\
8 \\
0 \\
\vdots \\
0\end{array}\right|$ & : & $\mid \begin{array}{l}\delta \\
\vdots \\
\vdots \\
\vdots \\
c\end{array}$ & 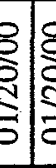 & $\begin{array}{l}8 \\
\stackrel{8}{8} \\
0 \\
\end{array}$ & 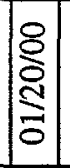 & & 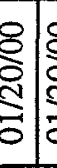 & $\begin{array}{c}8 \\
\text { s. } \\
\text { s. } \\
\end{array}$ & 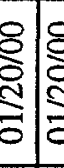 & & 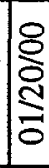 & | \\
\hline 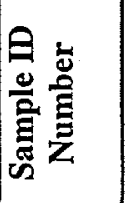 & $\mid \begin{array}{l}8 \\
0 \\
= \\
\\
0 \\
0 \\
8\end{array}$ & $\begin{array}{l}8 \\
0 \\
0 \\
0 \\
0 \\
0 \\
8\end{array}$ & $\begin{array}{l}8 \\
0 \\
2 \\
0 \\
0 \\
8 \\
8\end{array}$ & $\begin{array}{l}8 \\
8 \\
0 \\
0 \\
0 \\
08 \\
8\end{array}$ & 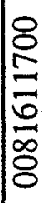 & $\mid \begin{array}{c}8 \\
0 \\
0 \\
0 \\
0 \\
0 \\
8 \\
8\end{array}$ & $\mid \begin{array}{l}8 \\
0 \\
0 \\
0 \\
0 \\
0 \\
8\end{array}$ & $\begin{array}{l}8 \\
0 \\
0 \\
6 \\
0 \\
0 \\
8\end{array}$ & $\mid \begin{array}{l}8 \\
0 \\
= \\
6 \\
0 \\
0 \\
8 \\
8\end{array}$ & $\frac{u}{z}$ & $\left|\begin{array}{c}8 \\
0 \\
0 \\
0 \\
0 \\
8 \\
8\end{array}\right|$ & $\begin{array}{l}\frac{8}{0} \\
\frac{7}{0} \\
\frac{0}{8} \\
8\end{array}$ & 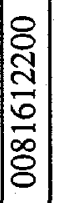 & 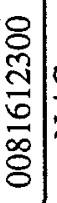 & 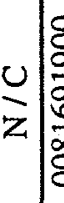 & 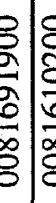 & $\begin{array}{l}8 \\
8 \\
0 \\
0 \\
0 \\
0 \\
8\end{array}$ & $\left|\begin{array}{c}8 \\
0 \\
0 \\
0 \\
0 \\
0 \\
8 \\
8\end{array}\right|$ & $\frac{u}{z} \mid$ & 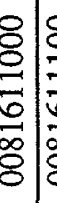 & $\begin{array}{l}8 \\
8 \\
\\
0 \\
8 \\
8 \\
\end{array}$ & 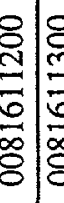 & $\frac{u}{z}$ & $\begin{array}{l}8 \\
8 \\
0 \\
0 \\
0 \\
8 \\
8\end{array}$ & $\mid \begin{array}{l}8 \\
0 \\
0 \\
0 \\
0 \\
0 \\
8\end{array}$ \\
\hline 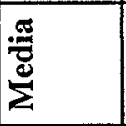 & is & $>$ & \& & $>$ & in & $>1$ & $\infty$ & $>$ & is & $>1$ & $\infty$ & $\infty$ & $\infty \mid$ & $\infty$ & $>0$ & s) & $>$ & $n$ & $>$ & $\infty 10$ & מ & $\infty 10$ & $n \mid>$ & as & $>$ \\
\hline 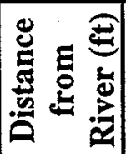 & & & 造 & $\underset{\infty}{\infty}$ & লি & ల్ & $\mid \begin{array}{l}8 \\
0 \\
2\end{array}$ & $\stackrel{8}{2}$ & 兽 & $\widetilde{్}$ & $\frac{8}{m}$ & $\underset{m}{\stackrel{8}{\circ}}$ & $\frac{8}{m}$ & $\frac{8}{8}$ & $\frac{8}{\mathrm{~m}}$ & 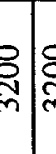 & ్రి & 0 & 0 & ్ㅣ & స్ర & శ్రి & & $\tilde{n}$ & 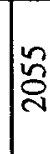 \\
\hline
\end{tabular}

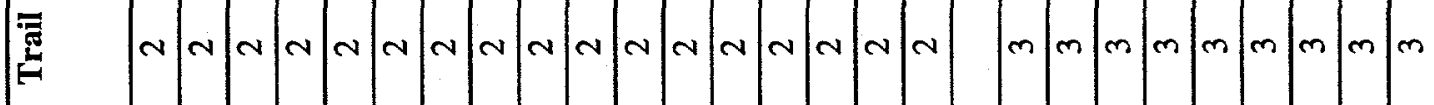


Table 4 -ciont.

\begin{tabular}{|c|c|c|c|c|c|c|c|c|c|c|c|c|}
\hline Trail & \begin{tabular}{|c|} 
Distance \\
from \\
River (ft)
\end{tabular} & Media & $\begin{array}{c}\text { Sample ID } \\
\text { Number }\end{array}$ & \begin{tabular}{|c|} 
Collection \\
Date
\end{tabular} & $\begin{array}{l}\text { Depth } \\
\text { (inch) }\end{array}$ & $\begin{array}{l}\text { Cs-137 } \\
(\mathrm{pCi} / \mathrm{g})\end{array}$ & $\begin{array}{l}\text { Cs-137 } \\
\text { Error }\end{array}$ & $\begin{array}{l}\mathrm{Co}-60 \\
(\mathrm{pCi} / \mathrm{g})\end{array}$ & $\begin{array}{l}\text { Co-60 } \\
\text { Error }\end{array}$ & $\begin{array}{c}\text { Sr-89,90 } \\
(\mathrm{pCi} / \mathrm{g})\end{array}$ & $\begin{array}{c}\text { Sr-89,90 } \\
\text { Error }\end{array}$ & \begin{tabular}{|c|} 
Gamma \\
Exposure \\
(mrem/d) \\
\end{tabular} \\
\hline 4 & 0 & $S$ & 0081628700 & $03 / 09 / 00$ & $0-3$ & $4.84 \mathrm{E}-02$ & $2.00 \mathrm{E}-02$ & $-2.85 \mathrm{E}-03$ & $2.07 \mathrm{E}-02$ & $\mathrm{~N} / \mathrm{A}$ & $\mathrm{N} / \mathrm{A}$ & 0.58 \\
\hline 4 & 0 & $\overline{\mathrm{V}}$ & $\mathrm{N} / \mathrm{C}$ & & & & & & & & & \\
\hline 4 & 960 & $\mathrm{~S}$ & 0081628800 & $03 / 09 / 00$ & $0-3$ & $6.36 \mathrm{E}+00$ & 3.37E-01 & $1.37 \mathrm{E}-03$ & $2.57 \mathrm{E}-02$ & $\mathrm{~N} / \mathrm{A}$ & $\mathrm{N} / \mathrm{A}$ & 0.58 \\
\hline 4 & 960 & $\mathrm{~V}$ & $\mathrm{~N} / \mathrm{C}$ & & & & & & & & & \\
\hline 4 & 1245 & $\mathrm{~S}$ & 0081628900 & $03 / 09 / 00$ & $0-3$ & $1.37 \mathrm{E}+01$ & $6.88 \mathrm{E}-01$ & $1.11 \mathrm{E}-02$ & $2.32 \mathrm{E}-02$ & $\mathrm{~N} / \mathrm{A}$ & $\mathrm{N} / \mathrm{A}$ & 0.62 \\
\hline 4 & 1245 & $\mathrm{~V}$ & $\mathrm{~N} / \mathrm{C}$ & & & & & & & & & \\
\hline 4 & 1690 & $S$ & 0081629000 & $03 / 09 / 00$ & $0-3$ & $1.72 \mathrm{E}+01$ & $8.61 \mathrm{E}-01$ & $9.37 \mathrm{E}-02$ & $2.72 \mathrm{E}-02$ & $\mathrm{~N} / \mathrm{A}$ & $\mathrm{N} / \mathrm{A}$ & 0.63 \\
\hline 4 & 1690 & $\mathrm{~V}$ & $\mathrm{~N} / \mathrm{C}$ & & & & & & & & & \\
\hline 4 & 1900 & $S$ & 0081629100 & $03 / 09 / 00$ & $0-3$ & $5.27 \mathrm{E}+01$ & $1.86 \mathrm{E}+00$ & $1.13 \mathrm{E}-01$ & $1.79 \mathrm{E}-02$ & $3.64 \mathrm{E}-01$ & $1.47 \mathrm{E}-01$ & 0.81 \\
\hline 4 & 1900 & $S$ & .0081629200 & $03 / 09 / 00$ & $3-6$ & $3.70 \mathrm{E}+01$ & $1.83 \mathrm{E}+00$ & $1.05 \mathrm{E}-01$ & $2.35 \mathrm{E}-02$ & $3.01 \mathrm{E}-01$ & $1.45 \mathrm{E}-01$ & \\
\hline 4 & 1900 & $S$ & 0081629300 & $03 / 09 / 00$ & $6-9$ & $1.32 \mathrm{E}+01$ & $6.62 \mathrm{E}-01$ & $1.49 \mathrm{E}-02$ & $2.15 \mathrm{E}-02$ & $1.19 \mathrm{E}-01$ & $1.75 \mathrm{E}-01$ & \\
\hline 4 & 1900 & $S$ & 0081629400 & $03 / 09 / 00$ & $9-12$ & $1.18 \mathrm{E}+01$ & $5.97 \mathrm{E}-01$ & $1.15 \mathrm{E}-02$ & $2.27 \mathrm{E}-02$ & $2.08 \mathrm{E}-01$ & $1.27 \mathrm{E}-01$ & \\
\hline 4 & 1900 & $\mathrm{~V}$ & $\mathrm{~N} / \mathrm{C}$ & & & & & & & & & \\
\hline 4 & 2390 & $S$ & 0081629500 & $03 / 09 / 00$ & $0-3$ & $2.03 \mathrm{E}-01$ & $3.56 \mathrm{E}-02$ & $1.51 \mathrm{E}-02$ & $1.91 \mathrm{E}-02$ & $\mathrm{~N} / \mathrm{A}$ & $\mathrm{N} / \mathrm{A}$ & 0.54 \\
\hline 4 & 2390 & $\mathrm{~V}$ & $\mathrm{~N} / \mathrm{C}$ & & & & & & & & & \\
\hline
\end{tabular}


Table 4 - cont.

\begin{tabular}{|c|c|c|c|c|c|c|c|c|c|c|c|c|}
\hline Trail & $\begin{array}{c}\text { Distance } \\
\text { from } \\
\text { River }(\mathrm{ft}) \\
\end{array}$ & Media & $\begin{array}{l}\text { Sample ID } \\
\text { Number }\end{array}$ & $\begin{array}{c}\text { Collection } \\
\text { Date }\end{array}$ & $\begin{array}{l}\text { Depth } \\
\text { (inch) }\end{array}$ & $\begin{array}{l}\text { Cs-137 } \\
(\mathrm{pCi} / \mathrm{g})\end{array}$ & $\begin{array}{l}\text { Cs-137 } \\
\text { Error }\end{array}$ & $\begin{array}{c}\mathrm{Co}-60 \\
(\mathrm{pCi} / \mathrm{g})\end{array}$ & $\begin{array}{l}\text { Co-60 } \\
\text { Error }\end{array}$ & $\begin{array}{c}\text { Sr-89,90 } \\
(\mathrm{pCi} / \mathrm{g})\end{array}$ & $\begin{array}{c}\text { Sr-89,90 } \\
\text { Error }\end{array}$ & \begin{tabular}{|c|} 
Gamma \\
Exposure \\
(mrem/d)
\end{tabular} \\
\hline 5 & 0 & $S$ & 0081616700 & $01 / 27 / 00$ & $0-3$ & $9.85 \mathrm{E}-02$ & $2.63 \mathrm{E}-02$ & $5.56 \mathrm{E}-02$ & $2.78 \mathrm{E}-02$ & $\mathrm{~N} / \mathrm{A}$ & $\bar{N} / \mathrm{A}$ & 0.34 \\
\hline 5 & 0 & $\bar{V}$ & 0081616800 & $01 / 27 / 00$ & & $-7.72 \mathrm{E}-03$ & $2.36 \mathrm{E}-02$ & $2.88 \mathrm{E}-02$ & $2.86 \mathrm{E}-02$ & $\mathrm{~N} / \mathrm{A}$ & $\overline{\mathrm{N} / \mathrm{A}}$ & \\
\hline 5 & 1750 & $S$ & 0081616900 & $01 / 27 / 00$ & $0-3$ & $8.03 E+00$ & $4.15 \mathrm{E}-01$ & $1.22 \mathrm{E}-02$ & $2.47 \mathrm{E}-02$ & $\mathrm{~N} / \mathrm{A}$ & N/A & 0.33 \\
\hline 5 & 1750 & $\bar{V}$ & $\mathrm{~N} / \mathrm{C}$ & & & & & & & & : & \\
\hline 5 & 1880 & $S$ & 0081617000 & $01 / 27 / 00$ & $0-3$ & $1.57 \mathrm{E}+01$ & $7.88 \mathrm{E}-01$ & $5.94 \mathrm{E}-02$ & $2.50 \mathrm{E}-02$ & N/A & $\mathrm{N} / \mathrm{A}$ & 0.37 \\
\hline 5 & 1880 & $\mathrm{~V}$ & $\mathrm{~N} / \mathrm{C}$ & & & & & & & & & \\
\hline 5 & 2100 & $S$ & 0081617100 & $01 / 27 / 00$ & $0-3$ & $4.90 \mathrm{E}+01$ & $2.42 \mathrm{E}-01$ & $1.57 \mathrm{E}-01$ & $3.00 \mathrm{E}-02$ & $2.81 \mathrm{E}-01$ & $1.85 \mathrm{E}-01$ & 0.71 \\
\hline 5 & 2100 & $S$ & 0081617200 & $01 / 27 / 00$ & $3-6$ & $3.80 \mathrm{E}+01$ & $1.88 \mathrm{E}+00$ & $1.32 \mathrm{E}-01$ & $4.52 \mathrm{E}-02$ & $7.41 \mathrm{E}-01$ & $2.15 \mathrm{E}-01$ & \\
\hline 5 & 2100 & $S$ & 0081617300 & $01 / 27 / 00$ & $6-9$ & $2.87 \mathrm{E}+01$ & $1.43 \mathrm{E}+00$ & $4.10 \mathrm{E}-02$ & $2.88 \mathrm{E}-02$ & $4.53 \mathrm{E}-01$ & $1.97 \mathrm{E}-01$ & \\
\hline 5 & 2100 & $S$ & 0081617400 & $01 / 27 / 00$ & $9-12$ & $2.27 \mathrm{E}+01$ & $1.13 \mathrm{E}+00$ & $7.18 \mathrm{E}-02$ & $2.72 \mathrm{E}-02$ & $1.21 \mathrm{E}-01$ & $1.73 \mathrm{E}-01$ & \\
\hline 5 & 2100 & $\mathrm{~V}$ & $\mathrm{~N} / \mathrm{C}$ & & & & & & & & & \\
\hline 5 & 2535 & $S$ & 0081617500 & $01 / 27 / 00$ & $0-3$ & $2.08 \mathrm{E}-01$ & $3.37 \mathrm{E}-02$ & $5.52 \mathrm{E}-03$ & $1.84 \mathrm{E}-02$ & $\mathrm{~N} / \mathrm{A}$ & $\mathrm{N} / \mathrm{A}$ & 0.31 \\
\hline 5 & 2535 & V & 0081617600 & $01 / 27 / 00$ & & $4.41 \mathrm{E}+00$ & $2.66 \mathrm{E}-01$ & $-3.33 \mathrm{E}-02$ & $3.22 \mathrm{E}-02$ & $\mathrm{~N} / \mathrm{A}$ & $\mathrm{N} / \mathrm{A}$ & \\
\hline & & & & & & & & & & & & \\
\hline 6 & 0 & $S$ & 0081628100 & $02 / 03 / 00$ & $0-3$ & $5.19 \mathrm{E}-01$ & $2.92 \mathrm{E}-02$ & $1.10 \mathrm{E}-02$ & $2.75 \mathrm{E}-02$ & $\mathrm{~N} / \mathrm{A}$ & $\mathrm{N} / \mathrm{A}$ & 0.33 \\
\hline 6 & 0 & $\mathrm{~V}$ & $\mathrm{~N} / \mathrm{C}$ & & & & & & & & & \\
\hline 6 & 1800 & $S$ & $\mathrm{~N} / \mathrm{C}$ & & $0-3$ & & & & & & & \\
\hline 6 & 1800 & $\bar{V}$ & $\mathrm{~N} / \mathrm{C}$ & & & & & & & & & \\
\hline 6 & 2300 & $\bar{S}$ & $\mathrm{~N} / \mathrm{C}$ & & $0-3$ & & & & & & & \\
\hline 6 & 2300 & $\mathrm{~V}$ & $\mathrm{~N} / \mathrm{C}$ & & & & & & & & & \\
\hline 6 & 2530 & $S$ & $\mathrm{~N} / \mathrm{C}$ & & $0-3$ & & & & & & & \\
\hline 6 & 2530 & $\bar{S}$ & $\mathrm{~N} / \mathrm{C}$ & & $3-6$ & & & & & & & \\
\hline 6 & 2530 & $S$ & $\mathrm{~N} / \mathrm{C}$ & & $6-9$ & & & & & & & \\
\hline 6 & 2530 & $S$ & $\mathrm{~N} / \mathrm{C}$ & & $9-12$ & & & & & & & \\
\hline 6 & 2530 & $\bar{V}$ & $\mathrm{~N} / \mathrm{C}$ & & & & & & & & & \\
\hline 6 & 2680 & $S$ & 0081628200 & $02 / 03 / 00$ & $0-3$ & $1.10 \mathrm{E}+00$ & $7.83 \mathrm{E}-02$ & $1.24 \mathrm{E}-02$ & $2.00 \mathrm{E}-02$ & N/A & N/A & Missing \\
\hline 6 & 2680 & $\mathrm{~V}$ & $\mathrm{~N} / \mathrm{C}$ & & & & & & & & & \\
\hline
\end{tabular}




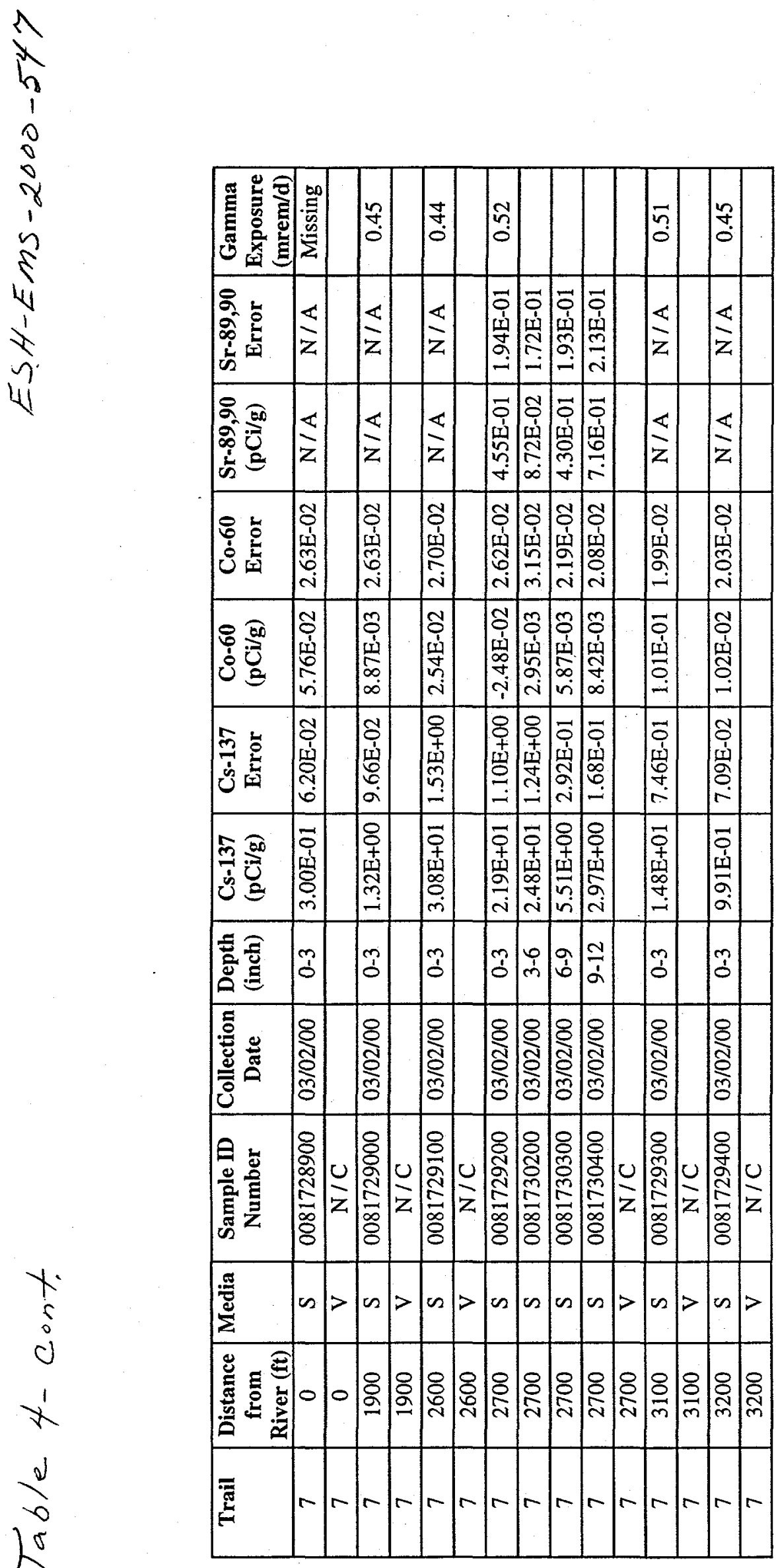


Tab/e 4 - cont.

\begin{tabular}{|c|c|c|c|c|c|c|c|c|c|c|c|c|}
\hline Trail & $\begin{array}{c}\text { Distance } \\
\text { from } \\
\text { River }(\mathrm{ft})\end{array}$ & \begin{tabular}{|l|} 
Media \\
\end{tabular} & $\begin{array}{c}\text { Sample ID } \\
\text { Number }\end{array}$ & $\begin{array}{c}\text { Collection } \\
\text { Date }\end{array}$ & $\begin{array}{l}\text { Depth } \\
\text { (inch) }\end{array}$ & $\begin{array}{l}\mathrm{Cs}-137 \\
(\mathrm{pCi} / \mathrm{g})\end{array}$ & $\begin{array}{l}\text { Cs-137 } \\
\text { Error }\end{array}$ & $\begin{array}{c}\mathrm{Co-60} \\
(\mathrm{pCi} / \mathrm{g})\end{array}$ & $\begin{array}{l}\text { Co-60 } \\
\text { Error }\end{array}$ & $\begin{array}{c}\mathrm{Sr}-89,90 \\
(\mathrm{pCi} / \mathrm{g})\end{array}$ & $\begin{array}{c}\text { Sr-89,90 } \\
\text { Error }\end{array}$ & $\begin{array}{c}\text { Gamma } \\
\text { Exposure } \\
\text { (mrem/d) }\end{array}$ \\
\hline 8 & 0 & $S$ & 0081729500 & $03 / 02 / 00$ & $0-3$ & $1.68 \mathrm{E}-01$ & $3.69 \mathrm{E}-02$ & $-2.19 E-02$ & $2.10 \mathrm{E}-02$ & $\mathrm{~N} / \mathrm{A}$ & $\mathrm{N} / \mathrm{A}$ & 0.43 \\
\hline 8 & 0 & $\mathrm{~V}$ & $\mathrm{~N} / \mathrm{C}$ & & & & & & & & & \\
\hline 8 & 550 & $S$ & 0081729600 & $03 / 02 / 00$ & $0-3$ & $7.46 \mathrm{E}-01$ & $5.72 \mathrm{E}-01$ & $3.13 \mathrm{E}-02$ & $2.31 \mathrm{E}-02$ & $\bar{N} / \mathrm{A}$ & $\mathrm{N} / \mathrm{A}$ & 0.47 \\
\hline 8 & 550 & $\mathrm{~V}$ & $\mathrm{~N} / \mathrm{C}$ & & & & & & & & & \\
\hline 8 & 915 & $\bar{S}$ & 0081729700 & $03 / 02 / 00$ & $0-3$ & $9.94 \mathrm{E}-01$ & $7.07 \mathrm{E}-02$ & $2.58 \mathrm{E}-02$ & $2.58 \mathrm{E}-02$ & $\mathrm{~N} / \mathrm{A}$ & N/A & 0.47 \\
\hline 8 & 915 & $\mathrm{~V}$ & $\mathrm{~N} / \mathrm{C}$ & & & & & & & & & \\
\hline 8 & 1460 & $S$ & 0081729800 & $03 / 02 / 00$ & $0-3$ & $1.22 \mathrm{E}+00$ & $8.35 \mathrm{E}-02$ & $5.21 \mathrm{E}-02$ & $2.83 \mathrm{E}-02$ & $\mathrm{~N} / \mathrm{A}$ & $\mathrm{N} / \mathrm{A}$ & 0.45 \\
\hline 8 & 1460 & $\mathrm{~V}$ & $\mathrm{~N} / \mathrm{C}$ & & & & & & & & & \\
\hline 8 & 2005 & $S$ & 0081729900 & $03 / 02 / 00$ & $0-3$ & $7.62 \mathrm{E}-01$ & $6.43 \mathrm{E}-02$ & $3.59 \mathrm{E}-03$ & $1.93 \mathrm{E}-02$ & $\mathrm{~N} / \mathrm{A}$ & N/A & 0.45 \\
\hline 8 & 2005 & $\mathrm{~V}$ & $\mathrm{~N} / \mathrm{C}$ & & & & & & & & & \\
\hline$\overline{8}$ & 2670 & $S$ & 0081730000 & $03 / 02 / 00$ & $0-3$ & $1.09 \mathrm{E}+01$ & $5.53 \mathrm{E}-01$ & $6.02 \mathrm{E}-03$ & $2.34 \mathrm{E}-02$ & $\mathrm{~N} / \mathrm{A}$ & N/A & 0.44 \\
\hline 8 & 2670 & $\bar{V}$ & $\mathrm{~N} / \mathrm{C}$ & & & & & & & & & \\
\hline 8 & 2900 & $S$ & $\overline{N / C}$ & & $0-3$ & & & & & & & \\
\hline 8 & 2900 & $S$ & $\mathrm{~N} / \mathrm{C}$ & & $3-6$ & & & & & & & \\
\hline 8 & 2900 & $S$ & $\overline{N / C}$ & & $6-9$ & & & & & & & \\
\hline 8 & 2900 & $S$ & $\mathrm{~N} / \mathrm{C}$ & & $9-12$ & & & & & & & \\
\hline 8 & 2900 & $\bar{V}$ & $\mathrm{~N} / \mathrm{C}$ & & & & & & & & & \\
\hline 8 & 3000 & $\bar{S}$ & 0081730100 & $03 / 02 / 00$ & $0-3$ & $3.48 \mathrm{E}-01$ & $4.24 \mathrm{E}-02$ & $6.95 \mathrm{E}-03$ & $1.99 \mathrm{E}-02$ & $\mathrm{~N} / \mathrm{A}$ & $\mathrm{N} / \mathrm{A}$ & 0.46 \\
\hline 8 & 3000 & $\mathrm{~V}$ & $\mathrm{~N} / \mathrm{C}$ & & & & & & & & & \\
\hline
\end{tabular}


Table 4 -cont.

\begin{tabular}{|c|c|c|c|c|c|c|c|c|c|c|c|c|}
\hline Trail & $\begin{array}{c}\text { Distance } \\
\text { from } \\
\text { River (ft) }\end{array}$ & Media & $\begin{array}{l}\text { Sample ID } \\
\text { Number }\end{array}$ & $\begin{array}{c}\text { Collection } \\
\text { Date }\end{array}$ & $\begin{array}{l}\text { Depth } \\
\text { (inch) }\end{array}$ & $\begin{array}{l}\mathrm{Cs}-137 \\
\text { (pCi/g) }\end{array}$ & $\begin{array}{l}\text { Cs-137 } \\
\text { Error }\end{array}$ & $\begin{array}{c}\mathrm{Co-60} \\
(\mathrm{pCi} / \mathrm{g})\end{array}$ & $\begin{array}{l}\text { Co-60 } \\
\text { Error }\end{array}$ & $\begin{array}{c}\text { Sr-89,90 } \\
(\mathrm{pCi} / \mathrm{g})\end{array}$ & $\begin{array}{c}\text { Sr-89,90 } \\
\text { Error }\end{array}$ & $\begin{array}{c}\text { Gamma } \\
\text { Exposure } \\
\text { (mrem/d) }\end{array}$ \\
\hline 9 & 0 & $\bar{S}$ & 0081638300 & $02 / 10 / 00$ & $0-3$ & $2.82 \mathrm{E}-01$ & $4.09 \mathrm{E}-02$ & $4.32 \mathrm{E}-02$ & $2.31 \mathrm{E}-02$ & $\mathrm{~N} / \mathrm{A}$ & N/A & 0.37 \\
\hline 9 & 0 & $\mathrm{~V}$ & $\mathrm{~N} / \mathrm{C}$ & & & & & & & & & \\
\hline 9 & 1680 & $S$ & 0081638400 & $02 / 10 / 00$ & $0-3$ & $1.63 \mathrm{E}+01$ & $8.24 \mathrm{E}-01$ & $2.87 \mathrm{E}-02$ & $2.81 \mathrm{E}-02$ & $\mathrm{~N} / \mathrm{A}$ & N/A & 0.44 \\
\hline 9 & 1680 & $\bar{V}$ & $\mathrm{~N} / \mathrm{C}$ & & & & & & & & & \\
\hline 9 & 2035 & $S$ & 0081638500 & $02 / 10 / 00$ & $0-3$ & $1.44 \mathrm{E}+01$ & $7.30 \mathrm{E}-01$ & $1.23 \mathrm{E}-02$ & $2.58 \mathrm{E}-02$ & $\mathrm{~N} / \mathrm{A}$ & $\mathrm{N} / \mathrm{A}$ & 0.43 \\
\hline 9 & 2035 & $\mathrm{~V}$ & $\mathrm{~N} / \mathrm{C}$ & & & & & & & & & \\
\hline 9 & 2200 & $S$ & 0081638600 & $02 / 10 / 00$ & $0-3$ & $3.06 \mathrm{E}+01$ & $1.51 \mathrm{E}+00$ & $4.16 \mathrm{E}-02$ & $2.45 \mathrm{E}-02$ & $1.51 \mathrm{E}-01$ & $1.74 \mathrm{E}-01$ & 0.46 \\
\hline 9 & 2200 & $S$ & $\mathrm{~N} / \mathrm{C}$ & & $3-6$ & & & & & & & \\
\hline 9 & 2200 & $S$ & $\mathrm{~N} / \mathrm{C}$ & & $6-9$ & & & & & & & \\
\hline 9 & 2200 & $S$ & $\mathrm{~N} / \mathrm{C}$ & & $9-12$ & & & & & & & \\
\hline 9 & 2200 & $\bar{V}$ & $\mathrm{~N} / \mathrm{C}$ & & & & & & & & & \\
\hline 9 & 2525 & $S$ & 0081638700 & $02 / 10 / 00$ & $0-3$ & $5.43 \mathrm{E}-01$ & $4.98 \mathrm{E}-02$ & $1.50 \mathrm{E}-03$ & $1.55 \mathrm{E}-02$ & $\mathrm{~N} / \mathrm{A}$ & N/A & 0.26 \\
\hline 9 & 2525 & $\mathrm{~V}$ & $\mathrm{~N} / \mathrm{C}$ & & & & & & & & & \\
\hline & & & & & & & & & & & & \\
\hline 10 & 0 & $S$ & 0081628400 & $02 / 03 / 00$ & $0-3$ & $5.07 \mathrm{E}+00$ & $2.77 \mathrm{E}-01$ & $3.88 \mathrm{E}-02$ & $2.58 \mathrm{E}-02$ & $\mathrm{~N} / \mathrm{A}$ & N/A & 0.33 \\
\hline 10 & 0 & $\mathrm{~V}$ & $\mathrm{~N} / \mathrm{C}$ & & & & & & & & & \\
\hline 10 & 100 & $S$ & 0081628500 & $02 / 03 / 00$ & $0-3$ & $9.60 \mathrm{E}+00$ & $4.92 \mathrm{E}-01$ & $4.03 \mathrm{E}-02$ & $2.42 \mathrm{E}-02$ & $1.95 \mathrm{E}-03$ & $1.65 \mathrm{E}-01$ & 0.39 \\
\hline 10 & 100 & $S$ & 0081638800 & $02 / 10 / 00$ & $3-6$ & $6.77 \mathrm{E}-01$ & $6.26 \mathrm{E}-02$ & $1.55 \mathrm{E}-03$ & $2.01 \mathrm{E}-02$ & $7.37 \mathrm{E}-01$ & $2.11 \mathrm{E}-01$ & \\
\hline 10 & 100 & $S$ & 0081638900 & $02 / 10 / 00$ & $6-9$ & $7.45 \mathrm{E}-01$ & $5.76 \mathrm{E}-02$ & $2.76 \mathrm{E}-02$ & $2.23 \mathrm{E}-02$ & $3.02 \mathrm{E}-01$ & $1.85 \mathrm{E}-01$ & \\
\hline 10 & 100 & $S$ & 0081639000 & $02 / 10 / 00$ & $9-12$ & $2.13 \mathrm{E}-01$ & $4.32 \mathrm{E}-02$ & $2.77 \mathrm{E}-03$ & $1.90 \mathrm{E}-02$ & $2.66 \mathrm{E}-01$ & $1.83 \mathrm{E}-01$ & \\
\hline 10 & 100 & $\mathrm{~V}$ & $\mathrm{~N} / \mathrm{C}$ & & & & & & & & & \\
\hline 10 & 240 & $S$ & 0081628600 & $02 / 03 / 00$ & $0-3$ & $7.16 \mathrm{E}-01$ & $5.73 \mathrm{E}-02$ & $-4.06 \mathrm{E}-03$ & $2.47 \mathrm{E}-02$ & N/A & $\mathrm{N} / \mathrm{A}$ & 0.30 \\
\hline
\end{tabular}

NOTES:

$S$ indicates soil

$\mathrm{V}$ indicates vegetation

N/C indicates not collected

N/A indicates not analyzed 